\title{
Hemihyperplasia-multiple lipomatosis syndrome
}

INSERM

\section{Source}

INSERM. (1999). Orphanet: an online rare disease and orphan drug data base. Hemihyperplasia-multiple lipomatosis syndrome. ORPHA:276280

Hemihyperplasia-multiple lipomatosis syndrome is a rare, genetic overgrowth syndrome characterized by non- progressive, asymmetrical, moderate hemihyperplasia (frequently affecting the limbs) associated with slow growing, painless, multiple, recurrent, subcutaneous lipomatous masses distributed throughout entire body (in particular back, torso, extremities, fingers, axillae). Superficial vascular malformations may also be associated. Increased risk of intra-abdominal embryonal malignancies may be associated. 